\title{
Obsessive-compulsive Personality Disorder: Etiologies, Impacts, and Treatments
}

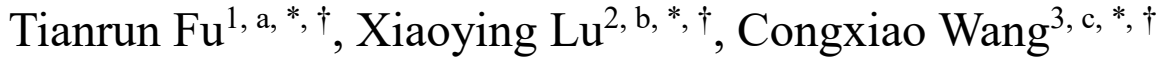 \\ ${ }^{1}$ Jiangxi Normal University, Nanchang, Jiangxi, China \\ ${ }^{2}$ Nanjing University of Posts and Telecommunications, Nanjing, Jiangsu, China \\ ${ }^{3}$ University of Michigan, Ann Arbor, Michigan, U.S. \\ "Corresponding author.Email: ${ }^{*}$ tianrun@jxnu.edu.cn, ${ }^{b}$ sami.lu@foxmail.com, ${ }^{c}$ congxiao@umich.edu, \\ These authors contributed equally.
}

\begin{abstract}
Obsessive-Compulsive Personality Disorder (OCPD) is one of the most prevalent personality disorders (PDs) in community and outpatient samples. It is characterized by a general pattern of concern with orderliness, perfectionism, excessive attention to details, mental and interpersonal control, and a need for control over one's environment at the expense of flexibility, openness, and efficiency. This article aimed at reviewing the literature on etiologies, impacts, and treatments of OCPD. Etiologies of OCPD are divided into two aspects, including psychoanalytic factors and biological factors, respectively. The impacts part shows both positive and negative impacts OCPD brings. There are two regular treatments of OCPD, which are medication and psychotherapies. The medication one offers anti-anxiety or antidepression medication like SSRIs, and the psychotherapies offer methods such as interpersonal therapy and cognitive therapy. This study can display an academic direction for future researchers.
\end{abstract}

Keywords: Obsessive-Compulsive personality Disorder, Etiology, Impacts, Treatment.

\section{INTRODUCTION}

Obsessive-compulsive personality disorder (OCPD) is one of ten personality disorders listed in the Diagnostic and Statistical Manual of Mental Disorders (fifth edition, DSM-5). It is characterized by an excessive preoccupation with orderliness, mental and interpersonal control, and perfectionism at the expense of efficiency, openness, and flexibility. In the International Classification of Diseases (tenth edition, ICD-10), OCPD is also called anankastic personality disorder. It is noted that Obsessive-compulsive personality disorder is one of the most prevalent personality disorders (PDs) in community and outpatient samples [1]. Epidemiological studies found that OCPD was the most prevalent PD among the general population [2], and it is the second most prevalent PD in inpatient samples (e.g., 28.3\%) [3]. In terms of co-morbidity, early studies found that the highest co-morbidity of OCPD with Cluster APD diagnoses and the most elevated odds ratios were found for the co-occurrence of OCPD with paranoid PD and schizoid PD [4-6].

Although the obsessive character is described hundred years ago in Freud's book, the anal character, surprisingly few studies have focused specifically on OCPD. Besides, as the most common personality disorder among the general population, it is associated with impairment in psychological function [7]. Considering the public health implications associated with OCPD traits, there is a relative dearth of empirical research on OCPD. The review aims to provide both practitioners and researchers with a summary of significant current theoretical developments and empirical findings regarding the etiology, impacts, and treatment of OCPD. Hopefully, this review can help future research conducted on OCPD, an important but often neglected personality disorder.

\section{METHODOLOGY}

The paper is a review based on literature collected using Web of Science and China National Knowledge Infrastructure. There are a total of 20 researches and studies cited. We put "Obsessive-Compulsive Personality Disorder" in the search engine for OCPD's basic information. "Obsessive-Compulsive Personality Disorder" and "Etiology" were put in the search engine for OCPD's etiologies. We search for "ObsessiveCompulsive Personality Disorder" and "Treatment" for 
the treatment part. The impacts part was based on papers about OCPD's impacts.

\section{THE ETIOLOGIES, IMPACTS, AND TREATMENTS OF OBSESSIVE- COMPULSIVE PERSONALITY DISORDER}

\subsection{Etiology}

Nowadays, more and more researchers study what causes OCPT. Generally, the etiologies of OCPD are divided into two ranges: psychoanalytic etiology and biological etiology.

In the aspect of psychoanalysis, researchers attributed obsessive character to parental strict disciplinary practices, parents' overprotection, and childhood traumatic experiences. Firstly, the obsessive character is hypothesized to be environmentally instilled through the mechanisms of parental over-control and strict disciplinary practices [8], which includes parents' control over toilet training. In Freud's early assumption on obsessive character, obsessive character arises from conflicts between parents and child over toilet training in the 2nd to 3rd year of life [9]. Besides, Nordahl and Stiles indicated that patients with obsessive-compulsive personality disorder reported experiencing significantly higher levels of parental overprotection and lower levels of paternal and maternal care compared to the healthy controls [10]. Thirdly, recent studies also found that childhood traumatic experiences, like emotional abuse, emotional neglect, physical abuse et al., might be risk factors in obsessive-compulsive personality disorder [11].

When it comes to the biological etiology, a majority of outcomes focus on heritability and brain structure. On the one hand, more recent studies, including twin studies, found evidence for heritable components for OCPD. Torgersen et al. reported the heritability to be 0.78 for OCPD and based on a meta-analysis of only three samples [12]. Aycicegi-Dinn, Dinn, and Caldwell-Harris said that personality traits are heritable based on modern personality theory and behavioral genetics research [13]. What is more, Hummelen et al. suggested that OCPD develops out of an intersubjective matrix where children with a moderate to the high inborn tendency of systemizing mechanism and thus displaying more rigidity, stubbornness, and perfectionism than average. Rigid and inflexible countermeasures meet them by parents who may share the same genetic disposition [5]. On the other hand, in terms of brain structure, Millon and Davis speculated a connection between obsessive character and limbic organization [14]. They hypothesized that the neurological regions of the limbic system might be dense among OCPD patients. Afterward, they suggested that there may be evolutionary underpinnings for such limbic differences.

\subsection{Impacts}

OCPD has some negative impacts on patients' routine work and relationships with other people. However, people with obsessive-compulsive traits also have some positive impacts in certain situations.

In terms of negative impacts, OCPD will affect behavior patterns, cognition and decision-making, and interpersonal relationship. In behavior patterns, patients with OCPD will attempt to control all areas of their environment by painstaking attention to rules, trivial details, and schedules. Tasks are performed with extreme caution and repeated checking for mistakes. Therefore, workaholism and miserliness are also often seen in those with this personality disorder. Besides, persons affected with this disorder may find it hard to relax, always feeling that time is running out for their activities and that more effort is needed to achieve their goals. Moreover, individuals with OCPD are mercilessly self-critical. Their thinking style is marked by cognitive distortions of dichotomous thinking, magnification, and catastrophizing [9]. In cognitive effect, recent studies are consistent with the theory that OCPD shares a similar range of executive dysfunction with compulsive disorders such as OCD in terms of cognitive inflexibility and impaired executive planning [15].

It should be noted that OCPD leads to less impairment than some PDs, such as schizotypal, avoidant, and borderline PD. Unlike patients with a clinical diagnosis of OCPD, those with obsessive-compulsive traits have some advantages in certain situations. In terms of cognition, those who have a healthy number of characteristics of OCPD will have the feeling of selfworth and acceptability, emotional stability, and relative resistance to stress [16]. Moreover, many obsessivecompulsive traits such as perseverance, industriousness, and self-control are highly regarded and rewarded within capitalistic, technological societies [9]. These traits are often perceived as positive characteristics in work.

\subsection{Treatments}

For people with Obsessive-Compulsive Personality Disorder (OCPD), medication and psychotherapies are regularly applied together for the most efficient outcomes. The treatment involves types of therapy, such as psychodynamic therapy. Individuals who can handle their determination and obsessive traits to assist their psychological growth are particularly successful. Medications can also be used to diminish symptoms of anxiety or depression. More researches focused on comorbid OCPD since they behaviors with more significant severity than simple OCPD group. However, the comorbid OCPD can behave differently with OCPD, and they show a much more solid tendency of getting depression or anxiety [17]. Thus, they should be treated with more anti-anxiety or anti-depression medication for 
the co-morbid OCPD group, like SSRIs. According to Pittenger, only using psychotherapy is just as effective as only using SSRIs in treating OCD, but both procedures are not entirely sufficient [18]. However, when SSRIs do not work, increasing psychological counseling may be helpful for the patients. When people feel like they reach the ceiling using only psychotherapy, adding SSRIs is not beneficial for further treatment. As OCD shares some common traits with OCPD, medications maybe not generally be considered the primary treatment for OCPD or OCD.

Traditionally, psychotherapies include cognitive therapy. Cognitive therapy may be useful for OCPD by addressing the irrationality of thoughts stemming from excessive conscientiousness, moralism, perfectionism, devotion to work, and stubbornness [19]. Besides the cognitive therapy, the researchers improved that into a metacognitive interpersonal therapy, which performs directing to OCPD patients or OCD patients. Metacognitive interpersonal therapy is an adopted version of dialectical behavior therapy, targeting patients' emotionally dysregulated, impulsive, or dramatic disorders. Moreover, a study focused on dialectical behaviour therapy working on the male group with OCPD, and the therapy was designed for reducing rigid thinking. It helps patients find a balance between their obsessive behaviours and coping with their lives [20]. The study reported that the patient had significant reductions. While some OCPD patients have difficulty with social functioning, schema therapy was invented as clarification-oriented psychotherapy, which helps patients renewal their social and occupational functioning [7]. During these psychotherapies, therapists and patients work together to understand motivations that may be unconscious and to eliminate root causes of interference. These therapies can target a larger scope of symptoms, like synchronous mood disorders. But, these therapies can be long-term, clients usually feel relief in the first few months, but it can take years for their deeper changes, such as the way they treat their behaviors.

\section{CONCLUSION AND FUTURE RESEARCH}

While OCPD is one of the most prevalent PDs in community studies, the paper outlines an overview of existing studies. It focuses on OCPD's etiology, impacts, and treatments.

The etiology of OCPD can be considered from psychoanalysis models and biological models. The psychoanalysis models focus on the environmental influences while the object growing up, like childhood trauma or parental abuse, can influence the chance of developing OCPD [11]. Moreover, for the biological models, researches targeted on genetics stated that OCPD could be heritable, and while some researches suggested that OCPD can be caused by abnormal brain structure for the limbic system. Researches of genetic models, including twin studies, found evidence for heritable components for OCPD. Nevertheless, the dataset does not support the view that specific genes are related to specific PDs Psychoanalytical and biological factors can contribute to OCPD. However, no evidence can explain the origins of the OCPD. So, there is a clear need for more studies into the etiology of OCPD to find compelling etiological accounts to understand the interplay between genes and environmental factors.

Then, for the impacts of OCPD on individuals, there are both advantages and disadvantages in a subjective view [9]. While OCPD can make people feel easier to be anxious about checking tasks, it can also bring perseverance. Meanwhile, OCPD is more minor impairment than other PDs on the cognition side.

Finally, the research presented several treatments in two fields. For psychotherapies, it is common to use interpersonal therapies and cognitive therapies. As for the medications, SSRIs are the prevalent choices. Traditionally, the best treatment combines both psychotherapies and medication. Although existing research has suggested various forms of psychotherapy that can effectively treat PDs, pharmacotherapy and psychotherapy research on OCPD as primary complaints are rare. The majority of studies involved patients with a primary Axis I disorder with co-morbid OCPD. Therefore, we do need more treatment studies on OCPD separately to clarify the core aspects of these treatment methods. Considering the prevalence of OCPD, the recommendation is that we should find an effective treatment method for OCPD in the future and have more empirical studies in the etiology of OCPD.

\section{REFERENCES}

[1] Grant, J. E., Mooney, M. E., \& Kushner, M. G. (2012). Prevalence, correlates, and comorbidity of DSM-IV obsessive-compulsive personality disorder: Results from the National Epidemiologic Survey on Alcohol and Related Conditions. Journal of Psychiatric Research, 46(4), 469-475. doi:10.1016/j.jpsychires.2012.01.009

[2] Mattia, J. I., \& Zimmerman, M. (2001). Epidemiology. In W. J. Livesley (Ed.). Handbook of personality disorders.theory, research, and treatment (pp. 107-124). New York: The Guilford Press.

[3] Rossi, A., Marinangeli, M. G., Butti, G., Kalyvoka, A., \& Petruzzi, C. (2000). Pattern of comorbidity among anxious and odd personality disorders: The case of obsessive-compulsive personality disorder. CNS Spectrums, 5, 23-26.

[4] Stuart, S., Pfohl, B., Battaglia, M., Bellodi, L., Grove, W., \& Cadoret, R. (1998). The co-occurence 
of DSM-III-R personality disorders. Journal of Personality Disorders, 12, 302-315.

[5] Hummelen, B., Wilberg, T., Pedersen, G., \& Karterud, S. (2008). The quality of the DSM-IV obsessive-compulsive personality disorder construct as a prototype category. The Journal of Nervous and Mental Disease, 196, 446-455.

[6] de Reus, R. J. M., \& Emmelkamp, P. M. G. (2012). Obsessive-compulsive personality disorder: a review of current empirical findings. Personality and Mental Health, 6(1), 1-21. doi:10.1002/pmh.144

[7] Diedrich, A., \& Voderholzer, U. (2015). ObsessiveCompulsive Personality Disorder: a Current Review. Current Psychiatry Reports, 17(2), 10. doi: 10.1007/s11920-014-0547-8

[8] Hertler, S. C. (2014). A Review and Critique of Obsessive-Compulsive Personality Disorder Etiologies: Reckoning With Heritability Estimates. Europe's Journal of Psychology, 10(1), 168-184. doi:10.5964/ejop.v10i1.679

[9] Villemarette-Pittman, N. R., Stanford, M. S., Greve, K. W., Houston, R. J., \& Mathias, C. W. (2004). Obsessive-compulsive personality disorder and behavioral disinhibition. Journal of Psychology, 138(1), 5-22. doi:10.3200/jrlp.138.1.5-22

[10] Nordahl, H. M., \& Stiles, T. C. (1997). Perceptions of parental bonding in patients with various personality disorders, lifetime depressive disorders, and healthy controls. Journal of personality disorders, 11(4), 391-402. doi:10.1521/pedi.1997.11.4.391

[11] Huang Li, Zhu Cuiying, Zhu Xiongzhao, Liu Wanting, Li Jiangxuan, Lei Hui. (2018) The Correlation between Obsessive-Compulsive Personality Disorder and Childhood Traumatic Experiences. Chineses Journal Of Clinical Psychology (05), 944-946+921.

[12] Torgersen, S., Lygren, S., Oien, P. A., Skre, I., Onstad, S., Edvardsen, J. Kringlen, E. (2000). A twin study of personality disorders. Comprehensive psychiatry, 41(6), 416-425. doi:10.1053/comp.2000.16560

[13] Aycicegi-Dinn, A., Dinn, W. M., \& CaldwellHarris, C. L. (2009). Obsessive-Compulsive Personality Traits: Compensatory Response to Executive Function Deficit? International Journal of Neuroscience, 119(4), 600-608. Doi: $10.1080 / 00207450802543783$

[14] Millon, T., \& Davis, R. D. (1996). Disorders of personality DSM-IV and beyond. New York, NY:
John Wiley \& Sons.Penke, L., Denissen, J. A., \& Miller, G. F. (2007). The evolutionary genetics of personality. European Journal of Personality, 21, 549-587. doi:10.1002/per.629

[15] Fineberg, N. A., Day, G. A., de Koenigswarter, N., Reghunandanan, S., Kolli, S., Jefferies-Sewell, K, Laws, K. R. (2015). The neuropsychology of obsessive-compulsive personality disorder: a new analysis. CNS Spectr, 20(5), 490-499. doi: $10.1017 /$ S1092852914000662

[16] Kline, P. (1967). Obsessional traits and emotional instability in a normal population. The British journal of medical psychology, 40(2), 153-157. doi:10.1111/j.2044-8341.1967.tb00563.x

[17] Sadri, S. K., McEvoy, P. M., Egan, S. J., Kane, R. T., Rees, C. S., \& Anderson, A. (2018). The relationship between obsessive compulsive personality and obsessive compulsive disorder treatment outcomes: Predictive utility and clinically significant change (vol 45, pg 524, 2017). Behavioural and Cognitive Psychotherapy, 46(6), 768-768. doi: 10.1017/s135246581800019x

[18] Pittenger, C., \& Bloch, M. H. (2014). Pharmacological Treatment of ObsessiveCompulsive Disorder. Psychiatric Clinics of North America, 37(3), 375. doi:10.1016/j.psc.2014.05.006

[19] Mancebo, M. C., Eisen, J. L., Grant, J. E., \& Rasmussen, S. A. (2005). Obsessive compulsive personality disorder and obsessive compulsive disorder: clinical characteristics, diagnostic difficulties, and treatment. Annals of clinical psychiatry official journal of the American Academy of Clinical Psychiatrists, 17(4), 197-204. doi: 10.1080/10401230500295305

[20] Lynch, T. R., \& Cheavens, J. S. (2008). Dialectical behavior therapy for comorbid personality disorders. Journal of Clinical Psychology, 64(2), 154-167. doi:10.1002/jclp.20449 